enormous mechanical movements under which, as the rocks sheared, the individual particles were forced over each other in one common direction, viz. from eastsouth-east to west-north-west. Further evidence of this mechanical movement is supplied by certain abundant fine parallel lines, like those of slickensides, which occur almost everywhere on the foliation-surfaces or other parallel divisional planes. They are especially well developed among the striped fissile schists and the gneissose flagstones. These lines run in the same general direction already mentioned (E.S.E. to W.N.W.). In many cases it may be observed that the component particles of the rocks are oriented in this same direction, while original quartz-veins are drawn out into parallel rods. Another important feature connected with these rocks is the development of minerals along the new planes of schistosity. In particular, the abundance of sericite mica is noteworthy, the longer axes of the crystals of which lic in a direction parallel with the slickenside-lines. Other micas, hornblende, actinolite, and garnets have also made their appearance along the same planes. This recrystallisation becomes more pronounced the further east one advances from the outcrop, or passes upwards from the great thrust-planc.

This accumulated evidence points to the conclusion that in the north-west of Sutherland the rocks have been powerfully affected by one grand series of terrestrial movements whereby new structures have been superinduced upon them. Among these changes the original characters of the rocks have been more or less completely effaced, and new crystalline structures have been produced. Although a normal upward succession from the Silurian strata into an overlying series of schists cannot be maintained in the north of Sutherland, it is nevertheless certain that the displacements and metamorphism here described are later than Lower Silurian time. It is also evident that these great changes had been completed before the timc of the Lower Old Red Sandstone, the conglomerates and breccias of which rest upon and are made up of fragments of the crystalline schists.

One final feature of the Durness and Eriboll area remains to be noticed. The geological structure of this region has been further complicated by the subsequent folding of the strata, and by a double system of normal faults ( $f f$ in Section) which affect the strata and thrust-planes alike. One set of normal faults trends north-north-east and southsouth-west, while another, which appears to be newer, trends more or less at right angles to these. By these two systems of later dislocations, the thrust-planes with their low hade have been intersected and shifted precisely as if they had been ordinary boundary-planes between two geological formations. Much of the difficulty, indeed, which has been from the first experienced in unravelling the complicated structure of this region may be traced to the effect of the intricate network of reversed and normal faulting. The very preservation of the Durness Basin is due to two normal step-faults, one of which lets down the quartzites more than $\mathrm{I} 200$ feet, while the other brings the whole Silurian Basin down to the sea-level.

B. N. PLACH JOHN HORNE

\section{THE GENESTS OF AN IDEA, OR STORY OF A DISCOVERY RELATING TO EQUATTONS IN MULTIPLE QUANTITY}

I VENTURE, even at the risk of appearing egoistical, to call the attention of a wider circle of English mathematical readers than are likely to notice it in the pages of the Comptes Renaius, to what appears to me a remarkable discovery in the theory of matrices, or, in other words, of multiple quantity which has lately presented itself to me. It seems to me the more necessary to do so because the nature of the theorem which constitutes the discovery would hardly be suspected from the leading title of the note in the Comptes Rendus in which it is contained, being indeed an after-thought, so that the sting of the paper has to be sought for in its tail.

Hamilton, of immortal memory, has given, in his "Lectures on Quaternions," a solution of a certain quadratic equation in quaternions, those algebraical entities which (building upon a suggestion in Prof. Cayley's ever-memorable paper ${ }^{1}$ on matrices, in the Philosophical Transactions for 1858 or thereabouts) I have, with the general concurrence of all who have given attention to the subject, found means of identifying with binary matrices or algebraical quantities of the second order, and this succeeded in determining the True Place of Quatcrnions in Nature. Now, what Hamilton has done for an equation of the second degree of quantities of the second order, the theorem in question effects in a much more simple and complete manner for a similar sort of equation of any degree and relating to quantities of any order.

The history of the discovery in question constitutes in itself, it seems to me, an interesting chapter in Heuristic. This is how it came about. Hamilton's equation admits of six solutions or roots, which arrange themselves naturally in three pairs, and stand in im. mediate, and what we algebraists call rational, relation to the three roots of a cubic equation, or rather to the six square roots of those three roots. From this it follows immediately that one single condition must be sufficient to reduce the number of distinct roots of the equation in quaternions or binary matrices from six to four, inasmuch as, when two roots of the cubic referred to become equal, two pairs of roots of the original equation must coincide. It naturally therefore became an object of interest to obtain the quantity which, equated to zero, expresses the condition of equality of two roots of this cubic, which of course may be effected by means of a well-known formula for finding the discriminant of a cubic equation; but the quantity so obtained directly from the cubic is of an exceedingly complex form, and leaves the mind entirely unsatisfied as to its true internal composition, just as from a handful of diamond dust it would be impossible to infer the crystalline form which constitutes the true idea, the raison or façon d'être of the glittering gem.

Again and again my mind reverted fruitlessly to the subject until, on September 28 last, pacing the deck of the splendid Dover and Calais boat, the Invicta, under the vivifying and genial rays of a bright and benignant sun, the idea occurred to me that the form to be determined must be subject to satisfy a certain partial differential equation, and without the aid of pen or pencil I succeeded, ere the voyage was half over, in identifying the discriminant of the cubic with that of a biquadratic of the simplest imaginable constitution possible: in technical language, supposing $p x^{2}+q x+r=0$ to be the equation in question, I discovered virtually that the desired discriminant is identical with that of the biquadratic form which is the determinant of the binary matrix (or the tensor squared of the quaternion) $p x^{2}+q x+r$ treated as if $x$ were an ordinary quantity. Starting from this point it was easy to infer all the possible cases of equality which could occur between the six roots; and, more than that, to classify under thirteen classes all the principal cases that could present themselves in the solution of the equation, not merely for the general case when there are six actual and determinate roots, but even for those cases when some of the roots pass off into infinity and become conceptual instead of actual, or else, without passing to infinity, remain actual but contain arbitrary constants.

This more-than-anticipated complete solution of the problem before me was in part suggested by the opening

"This paper constitutes a second birth of Algebra, its avatar in a new and sixtl volume of the American Mathematical Journ.xl. 
lincs of a memoir by M. Darboux on the solution of a biquadratic equation in Liouville's journal, with which its eminent author, my colleague in the Institute of France, providentially presented me shortly after my arrival in Paris, and which led me to see that the three pairs of solutions of the Hamiltonian equation must stand in immediate conceptual relation to the three pairs of sides of the complete quadrangle formed by a certain conic related to the form $p x^{2}+q x+r$ (in fact the determinant of the matrix $p u+q v+r w)$ with the fixed conic $v^{2}-u w$

Now comes the turning point, the àvayóptoss of this strange eventful history.

"There's a Divinity that shapes our ends,

Rough-hew them how we will." I

Seized with a sudden and fortunatc attack of bronchitis, which confined me to my bed, and in the access of nocturnal fever which that state induces, my thoughts reverted with increased activity to this geometrical figure. It became clear to my inner sense that there ought to be an immediate relation between the biquadratic determinant of the form $p x^{3}+q x+r$, spoken of above, and the three pairs of its roots, and seizing my courage with both hands, I made bold to declare to myself that the functional parts of the six identical equations to the six roots ought to be the three pairs of conjugate quadratic factors of the biquadratic in question.

But if this should turn out to be true, it became impossible not to suspect, or even more than half believe, that an analogous statement must admit of being made for a unilateral equation (i.e. one in which, as in Hamilton's, the multipliers of each power of the unknown matrix $x$ lie all on the same side (whether to the right or left) of it) whatever might be the degree of the equation, and whatever the order of the matrices concerned. In other words, supposing $f x=0$ to be such equation, and $\phi x=0$ to be the identical equation to any one of its roots, $\phi x$ ought to be contained as an algebraical factor in the determinant of the matrix $f x$ when, for the moment, $x$ therein is regarded as an ordinary quantity. If this were so, then the reciprocal theorem would neces sarily be true (on account of the determinant referred to being in gencral irreducible), viz. that, supposing $\omega$ to be the order of the matrices concerned, every algebraical divisor of it, say $\phi x$, of the degree $\omega$, must be the identically-zero function to one or the other of the matrices $x$ which satisfy the equation $f x=0$, and consequently it would be only necessary to combine, according to a well-known method of elimination, the given form $f x$ with each in succession of the derived forms, which constitute a brood or litter as it were, issuing " de son propre sein," to obtain all the roots of $f x$ by solving the ordinary algebraical equation det. $(f x)=0$, and that thus the solution of the unilateral equation would depend on the solution of an ordinary equation of the degree $n \omega, n$ being the degree of $f$ in $x$, and $\omega$ the order of the matrices concerned: the number of the roots of $f x$ would therefore be the number of ways of combining $n \omega$ things $\omega$ and $\omega$ together, i.e. $\frac{\Pi(n \omega)}{\Pi \omega \Pi(n-I) \omega}$. But herein arose a self-created difficulty, a phantasmal projection of my own brain, to block up the way, and throw doubt and discredit on all that precedes. Supposing $\omega=2$, the number of roots thus ascertained would be $\frac{2 n(2 n-1)}{2}$, or $2 n^{2}-n$, and for $n=3$ would be 15 . Now, in the London and Edinburgh Philosophical Magazine for May last, whilst $I$ had shown that $2 n^{2}-n$ is the number of roots of a unilateral equation in quaternions of the degree $n$, and of the trinomial or Jerrardian form, I thought I had proved the number of solutions of a complete cubic equation in quaternions to be 2 I (upon which I based the formula

I It is one of Descartes" "self-evident primary truths" that nothing which has happeneil could not have happened otherwise. $n\left(n^{2}-n+1\right)$ for a unilateral equation of quaternions of the degrce $n$. There was then the choice to be made - to abandon the conjectural theorem, or to admit an error in the supposed determination of the number $2 \mathrm{I}$. I felt no hesitation in making my election, especially as there was a loop-hole for error in such numerical determination, inasmuch as no actual arithmetical calculations had been made, but the order of a certain system of equations which ought to be equal to the number of roots of $f x$ was inferred from calculations in which all numerical quantities were left in blank ; it was therefore quite possible (however unexpected the fact) that some of the leading coefficients of the resolving equation of the degree 21 might become zero, ${ }^{1}$ and consequently that the order might fall below (although it could not rise above) that number. To my gratified surprise my faith met with its reward, for I soon found an easy proof of the remarkable theorem which I have ventured, in emulation of a phrase of Cauchy, to call a "pulcherrima regula," which will appear in the number of the Comptes Rendus next forthcoming after this date, and which may be summed up approximately in the following words:--Every latent root of every root of a given unilateral funition in matrices of any order, is an algebraical root of the determinant of that function taken as if the unknown were an ordinary quantity, and conversely every algebraical root of the determinant so taken is a latent root of one of the roots of the given function." This constitutes a marvellous extension (to a matrix implicitly given by a unilateral equation) of the already no-little-marvellous Hamilton-Cayley theorem of the identical equation to a matrix given explicitly.

My good genius met me on the deck of the Invicta, and only left me three weeks later on board the returning steamer from Boulogne. There my pleasing algebraical dream came to an end. ${ }^{3}$

New College, Oxford, October 26

\section{OUR FUTURE WATCHES AND CLOCKS}

H OWEVER long the use of the letters "a.m." and H "p.m." for distinguishing the two halves of the civil day may survive, it seems probable that the more rational method of counting the hours of the day continuously from midnight through twenty-four hours to the midnight following may before long come into use for a variety of purposes for which it is well adapted, even if it should not yet be generally employed. It seems proper, therefore, to consider in what way ordinary watches and clocks could be best accommodated to such a change in the mode of reckoning. To place twenty-four hours on one circle round the dial instead of twelve hours as at present seems the most natural change to make; but, in addition to a new dial, it would involve also some alteration in construction, since the hour hand would have to make one revolution only in the twenty-four hours instead of two. And there would be this further disadvantage, that the hours being more crowded together, the angular motion of the hand in moving through the space corresponding to one hour would be less-in fact, one-half of its present amount. It is to be remembered that, in taking time from a clock, persons probably pay small attention to the figures, either those for hours

'Or else that its functional part might be composite and throw off an irrelevant factor.

${ }^{2}$ In terms more precise as regards the converse the theorem runs as follows -The idintically-zero function to a root of $\mathrm{f} x$ is a factar of the determinani to $\mathrm{f}$, and conversely every factor of that determinant of degree equal to the order of $\mathrm{x}$ is identically zero.

3 A letter just received from M. Hermite informs me that M. Poincarré, in a paper presented ly him to the Institute on Monday last, takes up the wondrous tale of multiple quantity so largely treated of by me in recent articles in the Comptes Rendzrs. The subject could not be in better hands. The ball is served, and the most skilfut and practised players-the Cayleys, the Lipschitzes, the Poincarres, the Weyrs, the Buckheims (and who knows how many more?)-stand round ready to receive it, and keep it flying in the air.-November 8. 